\title{
Students' Perception of the Educational Environment in an Indian Physiotherapy College
}

\section{Prachita Walankar}

MGM College of Physiotherapy, prachita30@gmail.com

Vrushali Panhale

MGM College of Physiotherapy

Sayli Situt

MGM College of Physiotherapy

Follow this and additional works at: https://nsuworks.nova.edu/ijahsp

Part of the Medicine and Health Sciences Commons

\section{Recommended Citation}

Walankar P, Panhale V, Situt S. Students' Perception of the Educational Environment in an Indian Physiotherapy College. The Internet Journal of Allied Health Sciences and Practice. 2019 Jan 01;17(2), Article 1.

This Manuscript is brought to you for free and open access by the College of Health Care Sciences at NSUWorks. It has been accepted for inclusion in Internet Journal of Allied Health Sciences and Practice by an authorized editor of NSUWorks. For more information, please contact nsuworks@nova.edu. 


\title{
Students' Perception of the Educational Environment in an Indian Physiotherapy College
}

\begin{abstract}
Purpose: Educational environment has a significant impact on students' behavior, academic progress and quality of learning. The aim of this study was to evaluate the students' perception of their learning environment in an Indian physiotherapy college and compare their perceptions in terms of demographic attributes and academic level. Methods: A cross-sectional study was conducted among 420 undergraduate physiotherapy students. The Dundee Ready Education Environment Measure (DREEM), a 50-item, self-administered inventory was employed to assess the student's perception of learning environment. It comprises of five domains: students' perceptions of learning; students' perceptions of teachers; students' academic self-perceptions; students' perceptions of atmosphere and students' social self-perceptions. Descriptive statistics, t-test and analysis of variance (ANOVA) were used to analyze data. P-value < 0.05 was considered as significant. Results: The mean DREEM score was $122.66 \pm$ 17.39 reflecting a more of positive than negative educational environment in this institution. There was a significant difference in the overall DREEM score $(p=0.000)$ based on the year of study with highest mean score in first year $(127.57 \pm 13.81)$. Evaluating the sub-domains of perception, the students in all the years had a more positive perception of learning (29.22 \pm 5.35$)$, their perception of teachers moved in the right direction $(26.52 \pm 5.05)$, their academic self-perception was more on the positive side (21.23 \pm $3.72)$, they had a more positive perception of atmosphere (28.56 \pm 5.64$)$ and their social self-perception could be graded as not too bad $(17.13 \pm 3.08)$. No significant gender difference was noted for overall DREEM score and all domain scores. Conclusion: The present study revealed that all undergraduate physiotherapy students perceived their educational environment positively. However, some areas require remedial measures in order to enhance the educational experience. Advocating problem-based learning, student mentorship, counseling and workshops on teaching-learning for teachers might enable us to enrich our learning environment.
\end{abstract}

\section{Author Bio(s)}

1. Prachita Walankar, MPTh, is an Assistant Professor, Department of Musculoskeletal Sciences in MGM College of Physiotherapy, Kamothe, Navi Mumbai, India.

2. Vrushali Panhale, MPT is a Professor, Department of Musculoskeletal Sciences in MGM College of Physiotherapy, Kamothe, Navi Mumbai, India

3. Sayli Situt, BPTh, is an intern in MGM College of Physiotherapy, Kamothe, Navi Mumbai, India

\section{Acknowledgements}

We would like to thank all the students who participated in the study. 


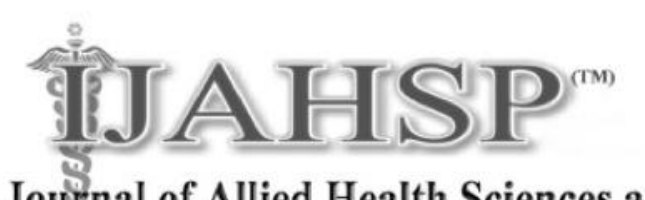

\title{
The Internet Journal of Allied Health Sciences and Practice \\ Dedicated to allied health professional practice and education
}

Vol. 17 No. 2 ISSN 1540-580X

\section{Students' Perceptions of the Educational Environment in an Indian Physiotherapy College.}

\author{
Prachita Walankar \\ Vrushali Panhale \\ Sayli Situt \\ MGM College of Physiotherapy \\ India
}

\begin{abstract}
Purpose: The educational environment has a significant impact on students' behavior, academic progress, and quality of learning. The aim of this study was to evaluate students' perceptions of their learning environment in an Indian physiotherapy college and compare their perceptions in terms of demographic attributes and academic level. Methods: A cross-sectional study was conducted among 420 undergraduate physiotherapy students. The Dundee Ready Education Environment Measure (DREEM), a 50-item self-administered inventory, was employed to assess the students' perceptions of the learning environment. It comprises five domains: students' perceptions of learning, students' perceptions of teachers, students' academic self-perceptions, students' perceptions of the atmosphere, and students' social self-perceptions. Descriptive statistics, t-test, and analysis of variance (ANOVA) were used to analyze data. A p-value of $<0.05$ was considered significant. Results: The mean DREEM score was $122.66 \pm 17.39$ reflecting a more positive than negative educational environment in this institution. There was a significant difference in the overall DREEM score $(p=0.000)$ based on the year of study with the highest mean score in the first year $(127.57$ $\pm 13.81)$. Evaluating the sub-domains of perception, the students in all years had a more positive perception of learning (29.22 \pm $5.35)$, their perception of teachers was that they were "moving in the right direction" $(26.52 \pm 5.05)$, their academic self-perception was more on the positive side $(21.23 \pm 3.72)$, they had a more positive perception of atmosphere $(28.56 \pm 5.64)$, and their social self-perception could be graded as "not too bad" $(17.13 \pm 3.08)$. No significant gender difference was noted for overall DREEM score and all domain scores. Conclusion: The present study revealed that all undergraduate physiotherapy students perceived their educational environment positively. However, some areas require remedial measures in order to enhance the educational experience. Advocating problem-based learning, student mentorship, counseling, and workshops on teaching-learning for teachers might enrich the learning environment.
\end{abstract}

Keywords: Educational Environment; Physiotherapy; DREEM instrument; Student perception; learning; teaching 


\section{INTRODUCTION}

An educational environment, a determinant of success in medical education, is defined as everything that happens within the classroom, department, or university. ${ }^{1}$ Evaluation of the learning environment is essential to maintain the quality standards of education as highlighted by the World Federation for Medical Education 1998.1-3 Among the medical educators, it is widely established that the academic and clinical environment has a great impact on students' attitudes, knowledge, skills, progression, and behaviors. ${ }^{1-2}$ The educational environment has been increasingly acknowledged as an essential factor for delivery of highquality medical education..$^{2-3}$ In health professional courses, it depends on interactions between different stakeholder groups and the organizational structure of the environment. There are three important aspects of the educational environment: physical climate, emotional climate, and intellectual climate. These, along with teaching and learning activities, are interrelated to promote satisfaction, effective learning, positive outcome, and progress among physiotherapy students. ${ }^{4}$ A positive institutional profile, improved student performance, higher staff morale, increased motivation among students, and quality teaching are viewed as some indicators of healthy educational environment. ${ }^{5}$ According to Harden et al, "measurement of [the] educational environment acts as a basis for the diagnosis of practices within an institution, and as the environment is changeable, the measurement may act as a platform for making necessary modifications for better educational practices in line with an institution's own goals". ${ }^{5}$ Evaluation of students' perception of learning helps the institute to ameliorate the strengths and address the weaknesses of the institution. 6

Physiotherapy education in India has evolved, like most other healthcare professions, from a diploma program to the current day status of degree and PhD programs. ${ }^{7}$ The 2-year diploma program progressed to a degree program; which extended the program from 2 years to $31 / 2$ years, and finally to the current 4 1/2-year program. The Bachelor of Physiotherapy (BPT) is a four-and-ahalf-year undergraduate program in physiotherapy with 6 months of mandatory clinical internship.

Physiotherapy students experience diverse learning activities within the environs of the physiotherapy college. Physiotherapy education is currently undergoing a transformation from a teacher-centered to a student-centered approach, where the teacher helps to facilitate learning by providing a healthy and positive learning environment. Students' perceptions about the educational climate play a crucial role in planning and implementing a curriculum. It also helps the stakeholders and the teaching faculty to reflect, reform, and remediate to make the curriculum student-friendly without compromising the standards and the quality of learning in the institute. Hence, systematic feedback and assessment are vital for successful management of the curriculum. ${ }^{8}$ The environment has an impact on students' learning experience, achievements, satisfaction, and success, which will help them to progress and achieve their goals. ${ }^{9}$ Assessment of educational environment will help to solve educational issues and improve the efficacy of education. Therefore, it is important to gather feedback from students regarding their experience in the learning environment.

A variety of assessment methods have been used to explore and quantify the substantial features of an educational environment, including qualitative, quantitative and mixed-methods. ${ }^{10-14}$ Many instruments with their own strengths and weaknesses are available to measure educational climate in professional healthcare education. The Dundee Ready Educational Environment Measure (DREEM) is the most widely used instrument to assess educational environment. ${ }^{15}$ The Dundee Ready Educational Environment Measure (DREEM) is a generic outcome measure used to analyze educational environment in healthcare professionals. ${ }^{10}$ The DREEM instrument has been found to be highly reliable in a variety of settings, which helps institutions to explore various aspects and identify weaknesses in order to formulate changes in curriculum. ${ }^{16}$ Therefore, the aim of the current study was to assess the perception of educational environment among undergraduate physiotherapy students in a private physiotherapy institute in India based on the DREEM inventory and determine the strengths and weakness of the institution.

\section{METHODS}

A cross-sectional study using a non-probability convenient sampling method was conducted among undergraduate physiotherapy students at a physiotherapy college in Mumbai, India. It offers a four and a half year, full-time undergraduate program that culminates in a professional qualification and a Bachelor of Physiotherapy degree. The study was approved by the Institutional Research Review Committee (Ref No. MGM/COP/IERC/111/2017).

The DREEM instrument was administered to undergraduate students among all levels - first, second, third, and fourth year and interns. The purpose of the study was explained to all students. The questionnaire was administered during the college working hours in the respective class. Students were asked not to reveal their identity while filling out the questionnaire. They were assured of confidentiality and anonymity. The completion of the DREEM inventory was undertaken on a voluntary basis, and written informed consent was obtained for participation from all the students. The DREEM instrument, a 50-item self-administered inventory, was used to assess educational environment. Each item is scored by respondents from 4 to 0 with a 5-point Likert response as follows: $4=$ strongly agree, $3=$ agree, $2=$ unsure, $1=$ disagree, and $0=$ strongly disagree. ${ }^{10,17}$ Among the questions,

(c) The Internet Journal of Allied Health Sciences and Practice, 2019 
nine items $(4,8,9,17,25,35,39,48$, and 50$)$ are negative and thus must be coded reversely. Items with a mean score of $\geq 3.5$ and higher are true positive points; those with a mean of $\leq 2$ are problematic areas and items with an average score of 2-3 indicate aspects of the environment that could be enhanced. The maximal overall score for the questionnaire is 200 . The global DREEM score is interpreted as follows: $0-50=$ very poor; $51-100=$ many problems; $101-150=$ more positive than negative; $151-200=$ excellent as per the interpretation guidelines provided by McAleer and Roff. 18,19

The items are divided into five broad categories based on the student's perception:

1) student's perception of learning (12 items with a maximum score of 48)

2) student's perception of teachers (11 items with a maximum score of 44)

3 ) student's perception academic self-perceptions ( 8 items with a maximum score of 32 )

4) student's perception of atmosphere (12 items with a maximum score of 48)

5 ) student's social self-perceptions ( 7 items with a maximum score of 28 )

The items of the DREEM inventory can be analyzed on the basis of three levels: individually, pooled into five subscales, and overall scoring.

\section{Statistical Analysis:}

The data was compiled in an MS Office Excel spreadsheet. Data were analyzed using Statistical Package for the Social Sciences (SPSS) version 24.00 for descriptive and inferential statistical analysis. Mean and standard deviations were calculated. Item-wise scores, total scores, and the domain scores for students of all years were calculated. One-way analysis of variance (ANOVA) with a post hoc Tukey test was used to identify the significant differences between the different years of study. An independent t-test was used to analyze gender differences. A p-value of $<0.05$ was considered statistically significant.

\section{RESULTS}

A total of 420 undergraduate physiotherapy students completed the DREEM inventory. The mean age of all participants was 20.11 \pm 1.63 years. In terms of gender, 370 participants $(88.09 \%)$ were female and $50(11.9 \%)$ were males. Table 1 demonstrates the demographic characteristics of the study population based on gender and year of study.

Table 1. Demographic profile of physiotherapy students based on gender and year of study.

\begin{tabular}{|l|l|l|l|l|l|l|}
\hline Variable & $\begin{array}{l}\text { First year } \\
(\mathbf{N}=\mathbf{8 8})\end{array}$ & $\begin{array}{l}\text { Second year } \\
(\mathbf{N}=\mathbf{8 7})\end{array}$ & $\begin{array}{l}\text { Third year } \\
(\mathbf{N}=\mathbf{6 9})\end{array}$ & $\begin{array}{l}\text { Fourth year } \\
(\mathrm{N}=83)\end{array}$ & $\begin{array}{l}\text { Interns } \\
(\mathbf{N}=93)\end{array}$ & $\begin{array}{l}\text { Total } \\
(\mathbf{N}=\mathbf{4 2 0})\end{array}$ \\
\hline $\begin{array}{l}\text { Age } \\
\text { (in years) }\end{array}$ & $18.03 \pm 0.3$ & $19.26 \pm 0.64$ & $19.89 \pm 0.69$ & $21.19 \pm 1.01$ & $22.08 \pm 0.70$ & $20.11 \pm 1.63$ \\
\hline Male & 12 & 13 & 7 & 9 & 9 & 50 \\
\hline Female & 76 & 74 & 62 & 74 & 84 & 370 \\
\hline
\end{tabular}

Mean scores of the subscales and overall DREEM score according to year of study are presented in Table 2. The overall DREEM score for this population was $122.66 \pm 17.39$ reflecting a more positive than negative educational environment in this institution. There was a significant difference in the overall DREEM score $(p=0.000)$ based on the year of study with the highest mean score for first years $(127.57 \pm 13.81)$ and lowest for interns (114.86 \pm 19.66$)$. Post hoc analysis revealed significant difference of interns with first years $(p=0.000)$, second years $(p=0.014)$ and fourth years $(p=0.000)$. Mean scores of the subscales and overall DREEM score according to gender are depicted in Table 3. No significant gender difference was noted for the overall DREEM score and all domain scores using an independent t-test.

Table 2. Comparison of mean subscale and overall DREEM scores based on year of study.

\begin{tabular}{|c|c|c|c|c|c|c|}
\hline Domain & $\begin{array}{c}\text { Students' } \\
\text { perception of } \\
\text { learning }\end{array}$ & $\begin{array}{c}\text { Students' } \\
\text { perception of } \\
\text { Teachers }\end{array}$ & $\begin{array}{c}\text { Students' } \\
\text { academic self- } \\
\text { perception }\end{array}$ & $\begin{array}{c}\text { Students' } \\
\text { perception of } \\
\text { Atmosphere }\end{array}$ & $\begin{array}{c}\text { Students' } \\
\text { social self- } \\
\text { perception }\end{array}$ & $\begin{array}{c}\text { Total DREEM } \\
\text { score }\end{array}$ \\
\hline First year & $31.13 \pm 3.72$ & $26.92 \pm 3.81$ & $21.96 \pm 2.88$ & $29.79 \pm 4.87$ & $17.75 \pm 2.52$ & $127.57 \pm 13.81$ \\
\hline $\begin{array}{c}\text { Second } \\
\text { year }\end{array}$ & $29.83 \pm 4.22$ & $25.85 \pm 3.88$ & $21.67 \pm 3.16$ & $27.86 \pm 5.27$ & $17.61 \pm 3.08$ & $122.82 \pm 14.55$ \\
\hline Third year & $28.39 \pm 5.27$ & $27 \pm 3.78$ & $20.33 \pm 3.78$ & $28.71 \pm 4.96$ & $16.54 \pm 3.13$ & $120.97 \pm 16.73$ \\
\hline
\end{tabular}




\begin{tabular}{|c|c|c|c|c|c|c|}
\hline $\begin{array}{c}\text { Fourth } \\
\text { year }\end{array}$ & $28.97 \pm 6.72$ & $32 \pm 0.00$ & $21.34 \pm 4.69$ & $28.2 \pm 6.44$ & $16.93 \pm 3.26$ & $127.45 \pm 18.28$ \\
\hline Interns & $27.66 \pm 5.73$ & $21.54 \pm 4.98$ & $20.69 \pm 3.76$ & $28.27 \pm 6.26$ & $16.71 \pm 3.25$ & $114.86 \pm 19.66$ \\
\hline Total & $29.22 \pm 5.35$ & $26.52 \pm 5.05$ & $21.23 \pm 3.72$ & $28.56 \pm 5.64$ & $17.13 \pm 3.08$ & $122.66 \pm 17.39$ \\
\hline P value & $0.000^{*}$ & $0.000^{*}$ & $0.03^{*}$ & 0.186 & $0.035^{*}$ & $0.000^{*}$ \\
\hline
\end{tabular}

Table 3. Comparison of mean domain and overall DREEM scores based on gender.

\begin{tabular}{|c|c|c|c|c|c|c|}
\hline Gender & $\begin{array}{c}\text { Students' } \\
\text { perception of } \\
\text { learning }\end{array}$ & $\begin{array}{c}\text { Students' } \\
\text { perception of } \\
\text { Teachers }\end{array}$ & $\begin{array}{c}\text { Students' } \\
\text { academic self- } \\
\text { perception }\end{array}$ & $\begin{array}{c}\text { Students' } \\
\text { perception of } \\
\text { Atmosphere }\end{array}$ & $\begin{array}{c}\text { Students' } \\
\text { social self- } \\
\text { perception }\end{array}$ & $\begin{array}{c}\text { Total DREEM } \\
\text { score }\end{array}$ \\
\hline Male & $29.18 \pm 4.96$ & $26.96 \pm 4.15$ & $21.04 \pm 3.65$ & $29.32 \pm 4.77$ & $16.96 \pm 3.39$ & $123.46 \pm 2.02$ \\
\hline Female & $29.22 \pm 5.4$ & $26.46 \pm 5.16$ & $21.25 \pm 3.74$ & $28.46 \pm 5.74$ & $17.15 \pm 3.04$ & $122.55 \pm 0.92$ \\
\hline P value & 0.959 & 0.516 & 0.703 & 0.313 & 0.681 & 0.730 \\
\hline
\end{tabular}

The majority of the students (85.47\%) perceived the educational environment as positive. 'A more positive perception' was observed among $78.57 \%$ of students in the domain of perception of learning. In the perception of teacher domain, $79.05 \%$ perceived that teachers were 'moving in the right direction'. The majority $(73.57 \%$ ) also had an academic self-perception that was 'more on the positive side'. Atmosphere was perceived as 'a more positive atmosphere' by $69.28 \%$, while $25.23 \%$ thought that there were issues that need changing. Nearly $59.04 \%$ thought that the society they live in was 'not too bad'. Overall, a level 3 score on all five domains was observed. The five sub-scales scores were a step below the ideal of the four-tiered scale (Table 4).

Table 4: Description of the overall DREEM score and five subscale scores of educational environment as reported by the students.

\begin{tabular}{|c|c|c|}
\hline DREEM Instrument & Interpretation & $\mathrm{N}(\%)$ \\
\hline \multicolumn{3}{|l|}{ Subscales } \\
\hline \multirow{4}{*}{$\begin{array}{l}\text { Students' perception of learning (SPL) } \\
\text { (12 items/maximum score 48) }\end{array}$} & $0-12$, very poor & $3(0.71 \%)$ \\
\hline & $13-24$, teaching is viewed negatively & $72(17.14 \%)$ \\
\hline & 25-36, a more positive approach & $330(78.57 \%)$ \\
\hline & $37-48$, teaching highly thought of & $15(3.57 \%)$ \\
\hline \multirow{4}{*}{$\begin{array}{l}\text { Students' perception of teachers (SPT) } \\
\text { (11 items/maximum score } 44 \text { ) }\end{array}$} & $0-11$, abysmal & $2(0.47 \%)$ \\
\hline & $12-22$, in need of some retraining & $75(17.85 \%)$ \\
\hline & $23-33$, moving in the right direction & $332(79.05 \%)$ \\
\hline & 34-44, model teachers & $11(2.61 \%)$ \\
\hline \multirow{4}{*}{$\begin{array}{l}\text { Students' academic self-perceptions(SASP) } \\
\text { (8 items/maximum score 32) }\end{array}$} & $0-8$, feeling of total failure & $1(0.23 \%)$ \\
\hline & $9-16$, many negative aspects & $37(8.8 \%)$ \\
\hline & 17-24, feeling more on the positive side & $309(73.57 \%)$ \\
\hline & 25-32, confident & $73(17.38 \%)$ \\
\hline \multirow{4}{*}{$\begin{array}{l}\text { Students' perception of atmosphere (SPA) } \\
\text { (12 items/maximum score 48) }\end{array}$} & $0-12$, a terrible environment & $5(1.19 \%)$ \\
\hline & $13-24$, there are many issues that need changing & $106(25.23 \%)$ \\
\hline & 25-36, a more positive atmosphere & $291(69.28 \%)$ \\
\hline & $37-48$, a good feeling overall & $18(4.28 \%)$ \\
\hline \multirow{4}{*}{$\begin{array}{l}\text { Students' social self-perceptions (SSSP) } \\
\text { (7 items/maximum score 28). }\end{array}$} & $0-7$, miserable & $3(0.71 \%)$ \\
\hline & $8-14$, not a nice place & $79(18.8 \%)$ \\
\hline & 15-21, not too bad & $248(59.04 \%)$ \\
\hline & $22-28$, very good socially & $79(18.8 \%)$ \\
\hline \multicolumn{3}{|l|}{ Overall score (out of 200) } \\
\hline $0-50$ & Very poor educational environment & $0(0)$ \\
\hline
\end{tabular}




\begin{tabular}{|l|l|r|}
\hline $51-100$ & Plenty of problems in the educational environment & $46(10.95 \%)$ \\
\hline $101-150$ & More positive than negative educational environment & $359(85.47 \%)$ \\
\hline $151-200$ & Excellent educational environment & $15(3.57 \%)$ \\
\hline
\end{tabular}

Table 5 shows the items with their average scores in different domains: 2 items scored above 3,39 items scored between 2 and 3 , and 9 items scored less than 2 . The two most highly rated items were 'I have good friends in this school' and 'My accommodation is pleasant'.

Table 5: Mean (SD) DREEM item scores for first, second, third, final year students and interns

\begin{tabular}{|c|c|c|c|c|c|c|c|c|}
\hline Domain & $\begin{array}{l}\text { Item } \\
\text { No. }\end{array}$ & Statement & $\begin{array}{l}\text { First } \\
\text { year } \\
(\mathrm{N}=88)\end{array}$ & $\begin{array}{l}\text { Second } \\
\text { year } \\
(\mathrm{N}=87)\end{array}$ & $\begin{array}{l}\text { Third } \\
\text { year } \\
(\mathrm{N}=69)\end{array}$ & $\begin{array}{l}\text { Fourth } \\
\text { year } \\
(\mathrm{N}=83)\end{array}$ & $\begin{array}{l}\text { Interns } \\
(N=93)\end{array}$ & $\begin{array}{l}\text { Total } \\
(\mathrm{N}= \\
420)\end{array}$ \\
\hline \multirow[t]{12}{*}{ SPL } & 1 & $\begin{array}{l}\text { I am encouraged to participate } \\
\text { in teaching sessions }\end{array}$ & $\begin{array}{l}2.95 \pm \\
0.67\end{array}$ & $\begin{array}{l}2.74 \\
\pm 0.89\end{array}$ & $\begin{array}{l}2.73 \\
\pm 0.65\end{array}$ & $\begin{array}{l}2.78 \pm 0.7 \\
6\end{array}$ & $\begin{array}{l}2.66 \\
\pm 0.71\end{array}$ & $\begin{array}{l}2.77 \\
\pm 0.75\end{array}$ \\
\hline & 7 & $\begin{array}{l}\text { The teaching is often } \\
\text { stimulating }\end{array}$ & $\begin{array}{l}2.72 \\
\pm 0.78\end{array}$ & $\begin{array}{l}2.55 \pm \\
0.83\end{array}$ & $\begin{array}{l}2.47 \pm \\
0.86\end{array}$ & $\begin{array}{l}2.56 \\
\pm 0.87\end{array}$ & $\begin{array}{l}2.24 \\
\pm 0.96\end{array}$ & $\begin{array}{l}2.51 \pm \\
0.87\end{array}$ \\
\hline & 13 & $\begin{array}{l}\text { The teaching is registrar } \\
\text { centered }\end{array}$ & $\begin{array}{l}2.73 \pm \\
0.83\end{array}$ & $\begin{array}{l}2.45 \\
\pm 0.89\end{array}$ & $\begin{array}{l}2.24 \\
\pm 1.02\end{array}$ & $\begin{array}{l}2.50 \pm 1.0 \\
1\end{array}$ & $\begin{array}{l}2.01 \\
\pm 1.02\end{array}$ & $\begin{array}{l}2.39 \\
\pm 0.98\end{array}$ \\
\hline & 16 & $\begin{array}{l}\text { The teaching helps to develop } \\
\text { my competence }\end{array}$ & $\begin{array}{l}2.77 \pm \\
1.00\end{array}$ & $\begin{array}{l}2.79 \\
\pm 0.74\end{array}$ & $\begin{array}{l}2.84 \\
\pm 0.71\end{array}$ & $\begin{array}{l}2.61 \\
\pm 0.98\end{array}$ & $\begin{array}{l}2.61 \pm \\
1.01\end{array}$ & $\begin{array}{l}2.72 \pm \\
0.91\end{array}$ \\
\hline & 20 & The teaching is well focused & $\begin{array}{l}2.73 \\
\pm 0.79\end{array}$ & $\begin{array}{l}2.67 \pm \\
0.79\end{array}$ & $\begin{array}{l}2.46 \\
\pm 0.97\end{array}$ & $\begin{array}{l}2.59 \\
\pm 1.00\end{array}$ & $\begin{array}{l}2.39 \\
\pm 0.95\end{array}$ & $\begin{array}{l}2.57 \\
\pm 0.91\end{array}$ \\
\hline & 21 & $\begin{array}{l}\text { The teaching helps to develop } \\
\text { my confidence }\end{array}$ & $\begin{array}{l}2.76 \pm \\
0.80\end{array}$ & $\begin{array}{l}2.73 \\
\pm 0.90\end{array}$ & $\begin{array}{l}2.26 \\
\pm 0.79\end{array}$ & $\begin{array}{l}2.49 \pm \\
1.00\end{array}$ & $\begin{array}{l}2.46 \\
\pm 0.95\end{array}$ & $\begin{array}{l}2.55 \\
\pm 0.91\end{array}$ \\
\hline & 24 & $\begin{array}{l}\text { The teaching time is put to } \\
\text { good use }\end{array}$ & $\begin{array}{l}2.45 \pm \\
0.82\end{array}$ & $\begin{array}{l}2.02 \\
\pm 1.10\end{array}$ & $\begin{array}{l}1.79 \pm \\
1.09\end{array}$ & $\begin{array}{l}2.36 \\
\pm 1.09\end{array}$ & $\begin{array}{l}2.23 \\
\pm 1.09\end{array}$ & $\begin{array}{l}2.19 \\
\pm 1.06\end{array}$ \\
\hline & 25 & $\begin{array}{l}\text { The teaching over emphasizes } \\
\text { factual learning }\end{array}$ & $\begin{array}{l}1.69 \\
\pm 0.93\end{array}$ & $\begin{array}{l}1.66 \\
\pm 0.87\end{array}$ & $\begin{array}{l}1.68 \pm \\
0.81\end{array}$ & $\begin{array}{l}1.42 \pm \\
0.98\end{array}$ & $\begin{array}{l}1.84 \\
\pm 0.92\end{array}$ & $\begin{array}{l}1.66 \pm \\
0.91\end{array}$ \\
\hline & 38 & $\begin{array}{l}\text { I am clear about the learning } \\
\text { objectives of the course }\end{array}$ & $\begin{array}{l}3.03 \\
\pm 0.70\end{array}$ & $\begin{array}{l}2.81 \pm \\
0.70\end{array}$ & $\begin{array}{l}2.75 \\
\pm 0.81\end{array}$ & $\begin{array}{l}2.74 \\
\pm 0.85\end{array}$ & $\begin{array}{l}2.73 \\
\pm 0.92\end{array}$ & $\begin{array}{l}2.81 \\
\pm 0.80\end{array}$ \\
\hline & 44 & $\begin{array}{l}\text { The teaching encourages me } \\
\text { to be an active learner }\end{array}$ & $\begin{array}{l}2.68 \pm \\
0.83\end{array}$ & $\begin{array}{l}2.77 \\
\pm 0.77\end{array}$ & $\begin{array}{l}2.60 \\
\pm 0.73\end{array}$ & $\begin{array}{l}2.37 \\
\pm 0.98\end{array}$ & $\begin{array}{l}2.29 \\
\pm 1.01\end{array}$ & $\begin{array}{l}2.54 \\
\pm 0.89\end{array}$ \\
\hline & 47 & $\begin{array}{l}\text { Long-term learning is } \\
\text { emphasized over short-term } \\
\text { learning }\end{array}$ & $\begin{array}{l}3.04 \pm \\
0.74\end{array}$ & $\begin{array}{l}2.79 \\
\pm 1.01\end{array}$ & $\begin{array}{l}2.56 \\
\pm 0.81\end{array}$ & $\begin{array}{l}2.81 \pm \\
1.06\end{array}$ & $\begin{array}{l}2.72 \pm \\
0.82\end{array}$ & $\begin{array}{l}2.79 \\
\pm 0.90\end{array}$ \\
\hline & 48 & $\begin{array}{l}\text { The teaching is too teacher } \\
\text { centered }\end{array}$ & $\begin{array}{l}1.53 \pm \\
0.78\end{array}$ & $\begin{array}{l}1.79 \pm \\
0.95\end{array}$ & $\begin{array}{l}1.95 \\
\pm 0.86\end{array}$ & $\begin{array}{l}1.69 \pm \\
0.98\end{array}$ & $\begin{array}{l}1.43 \pm \\
0.93\end{array}$ & $\begin{array}{l}1.66 \pm \\
0.92\end{array}$ \\
\hline \multirow[t]{4}{*}{ SPT } & 2 & $\begin{array}{l}\text { The teachers are } \\
\text { knowledgeable }\end{array}$ & $\begin{array}{l}2.95 \pm \\
0.52\end{array}$ & $\begin{array}{l}3.05 \\
\pm 0.49\end{array}$ & $\begin{array}{l}2.91 \\
\pm 0.65\end{array}$ & $\begin{array}{l}3.01 \\
\pm 0.57\end{array}$ & $\begin{array}{l}2.78 \pm \\
0.65\end{array}$ & $\begin{array}{l}2.94 \\
\pm 0.58\end{array}$ \\
\hline & 6 & $\begin{array}{l}\text { The teachers espouse a } \\
\text { patient-centered approach to } \\
\text { consulting }\end{array}$ & $\begin{array}{l}2.87 \\
\pm 0.67\end{array}$ & $\begin{array}{l}2.82 \\
\pm 0.75\end{array}$ & $\begin{array}{l}2.84 \\
\pm 0.69\end{array}$ & $\begin{array}{l}2.63 \pm \\
0.98\end{array}$ & $\begin{array}{l}2.46 \pm \\
0.87\end{array}$ & $\begin{array}{l}2.72 \\
\pm 0.82\end{array}$ \\
\hline & 8 & $\begin{array}{l}\text { The teachers ridicule their } \\
\text { students }\end{array}$ & $\begin{array}{l}1.57 \\
\pm 0.94\end{array}$ & $\begin{array}{l}1.85 \\
\pm 1.17\end{array}$ & $\begin{array}{l}2.11 \\
\pm 0.91\end{array}$ & $\begin{array}{l}1.77 \pm \\
1.02\end{array}$ & $\begin{array}{l}1.56 \pm \\
1.06\end{array}$ & $\begin{array}{l}1.75 \pm \\
1.04\end{array}$ \\
\hline & 9 & The teachers are authoritarian & $\begin{array}{l}1.19 \pm \\
0.86\end{array}$ & $\begin{array}{l}1.24 \\
\pm 0.95\end{array}$ & $\begin{array}{l}1.33 \pm \\
0.85\end{array}$ & $\begin{array}{l}1.14 \\
\pm 0.76\end{array}$ & $\begin{array}{l}1.34 \\
\pm 1.01\end{array}$ & $\begin{array}{l}1.25 \\
\pm 0.89\end{array}$ \\
\hline
\end{tabular}




\begin{tabular}{|c|c|c|c|c|c|c|c|c|}
\hline & 18 & $\begin{array}{l}\text { The teachers have good } \\
\text { communication skills with } \\
\text { patients }\end{array}$ & $\begin{array}{l}3.13 \\
\pm 0.73\end{array}$ & $\begin{array}{l}3.01 \\
\pm 0.73\end{array}$ & $\begin{array}{l}2.92 \\
\pm 0.64\end{array}$ & $\begin{array}{l}3.01 \pm \\
0.78\end{array}$ & $\begin{array}{l}2.55 \\
\pm 0.90\end{array}$ & $\begin{array}{l}2.92 \\
\pm 0.79\end{array}$ \\
\hline & 29 & $\begin{array}{l}\text { The teachers are good at } \\
\text { providing feedback to students }\end{array}$ & $\begin{array}{l}2.45 \\
\pm 1.07\end{array}$ & $\begin{array}{l}2.26 \pm \\
1.02\end{array}$ & $\begin{array}{l}2.15 \\
\pm 0.97\end{array}$ & $\begin{array}{l}2.44 \\
\pm 1.01\end{array}$ & $\begin{array}{l}2.16 \\
\pm 0.97\end{array}$ & $\begin{array}{l}2.30 \pm \\
1.01\end{array}$ \\
\hline & 32 & $\begin{array}{l}\text { The teachers provide } \\
\text { constructive criticism here }\end{array}$ & $\begin{array}{l}2.76 \pm \\
0.77\end{array}$ & $\begin{array}{l}2.39 \\
\pm 0.94\end{array}$ & $\begin{array}{l}2.57 \\
\pm 0.82\end{array}$ & $\begin{array}{l}2.43 \\
\pm 0.95\end{array}$ & $\begin{array}{l}2.38 \pm \\
1.07\end{array}$ & $\begin{array}{l}2.50 \pm \\
0.93\end{array}$ \\
\hline & 37 & $\begin{array}{l}\text { The teachers give clear } \\
\text { examples }\end{array}$ & $\begin{array}{l}2.76 \\
\pm 0.80\end{array}$ & $\begin{array}{l}2.62 \pm \\
0.89\end{array}$ & $\begin{array}{l}2.72 \\
\pm 0.72\end{array}$ & $\begin{array}{l}2.65 \\
\pm 0.96\end{array}$ & $\begin{array}{l}2.41 \\
\pm 0.91\end{array}$ & $\begin{array}{l}2.62 \\
\pm 0.87\end{array}$ \\
\hline & 39 & $\begin{array}{l}\text { The teachers get angry in } \\
\text { teaching sessions }\end{array}$ & $\begin{array}{l}1.76 \\
\pm 1.00 \\
\end{array}$ & $\begin{array}{l}1.50 \pm \\
1.03\end{array}$ & $\begin{array}{l}2.13 \pm \\
1.06\end{array}$ & $\begin{array}{l}2.19 \pm \\
1.10\end{array}$ & $\begin{array}{l}1.64 \pm \\
1.12\end{array}$ & $\begin{array}{l}1.82 \pm \\
1.09\end{array}$ \\
\hline & 40 & $\begin{array}{l}\text { The teachers are well prepared } \\
\text { for their teaching sessions }\end{array}$ & $\begin{array}{l}2.65 \pm \\
0.90\end{array}$ & $\begin{array}{l}2.81 \pm \\
0.77\end{array}$ & $\begin{array}{l}2.55 \pm \\
0.91\end{array}$ & $\begin{array}{l}2.84 \pm \\
0.77\end{array}$ & $\begin{array}{l}2.20 \pm \\
0.99\end{array}$ & $\begin{array}{l}2.60 \pm \\
0.90\end{array}$ \\
\hline & 49 & $\begin{array}{l}\text { The students irritate the } \\
\text { teachers }\end{array}$ & $\begin{array}{l}2.78 \pm \\
0.85\end{array}$ & $\begin{array}{l}2.26 \pm \\
1.05\end{array}$ & $\begin{array}{l}2.72 \pm \\
0.70\end{array}$ & $\begin{array}{l}2.69 \\
\pm 1.13\end{array}$ & $\begin{array}{l}2.34 \\
\pm 0.99\end{array}$ & $\begin{array}{l}2.55 \pm \\
0.98\end{array}$ \\
\hline & & & & & & & & \\
\hline \multirow[t]{8}{*}{ SASP } & 5 & $\begin{array}{l}\text { Learning strategies which } \\
\text { worked for me before continue } \\
\text { to work for }\end{array}$ & $\begin{array}{l}2.77 \pm \\
0.69\end{array}$ & $\begin{array}{l}2.59 \pm \\
0.84\end{array}$ & $\begin{array}{l}2.36 \\
\pm 0.85\end{array}$ & $\begin{array}{l}2.51 \\
\pm 1.05\end{array}$ & $\begin{array}{l}2.55 \\
\pm 0.84\end{array}$ & $\begin{array}{l}2.57 \pm \\
0.86\end{array}$ \\
\hline & 10 & $\begin{array}{l}\text { I am confident about passing } \\
\text { this year }\end{array}$ & $\begin{array}{l}2.78 \pm \\
0.83\end{array}$ & $\begin{array}{l}2.89 \pm \\
0.87\end{array}$ & $\begin{array}{l}2.73 \\
\pm 0.81\end{array}$ & $\begin{array}{l}2.89 \\
\pm 0.76\end{array}$ & $\begin{array}{l}3.08 \pm \\
0.88\end{array}$ & $\begin{array}{l}2.88 \\
\pm 0.84\end{array}$ \\
\hline & 22 & $\begin{array}{l}\text { I feel I am being well prepared } \\
\text { for my profession }\end{array}$ & $\begin{array}{l}2.81 \\
\pm 0.78\end{array}$ & $\begin{array}{l}2.73 \pm \\
0.78\end{array}$ & $\begin{array}{l}2.36 \pm \\
0.80\end{array}$ & $\begin{array}{l}2.66 \\
\pm 1.05\end{array}$ & $\begin{array}{l}2.34 \pm \\
0.98\end{array}$ & $\begin{array}{l}2.59 \pm \\
0.90\end{array}$ \\
\hline & 26 & $\begin{array}{l}\text { Last year's work has been a } \\
\text { good preparation for this year's } \\
\text { work }\end{array}$ & $\begin{array}{l}2.61 \pm \\
0.77\end{array}$ & $\begin{array}{l}2.74 \pm \\
0.87\end{array}$ & $\begin{array}{l}2.49 \pm \\
0.86\end{array}$ & $\begin{array}{l}2.56 \\
\pm 1.02\end{array}$ & $\begin{array}{l}2.48 \pm \\
0.84\end{array}$ & $\begin{array}{l}2.58 \\
\pm 0.88\end{array}$ \\
\hline & 27 & $\begin{array}{l}\text { I am able to memorize all I } \\
\text { need }\end{array}$ & $\begin{array}{l}2.62 \pm \\
0.99\end{array}$ & $\begin{array}{l}2.27 \pm \\
1.07\end{array}$ & $\begin{array}{l}2.49 \pm \\
0.86\end{array}$ & $\begin{array}{l}2.56 \pm \\
1.02\end{array}$ & $\begin{array}{l}2.48 \pm \\
0.84\end{array}$ & $\begin{array}{l}2.58 \\
\pm 0.88\end{array}$ \\
\hline & 31 & $\begin{array}{l}\text { I have learned a lot about } \\
\text { empathy in my profession }\end{array}$ & $\begin{array}{l}2.86 \pm \\
0.87\end{array}$ & $\begin{array}{l}2.95 \pm \\
0.72\end{array}$ & $\begin{array}{l}2.75 \pm \\
0.84\end{array}$ & $\begin{array}{l}2.90 \pm \\
0.83\end{array}$ & $\begin{array}{l}2.70 \pm \\
0.92\end{array}$ & $\begin{array}{l}2.83 \pm \\
0.84\end{array}$ \\
\hline & 41 & $\begin{array}{l}\text { My problem-solving skills are } \\
\text { being well developed here }\end{array}$ & $\begin{array}{l}2.51 \pm \\
0.93\end{array}$ & $\begin{array}{l}2.45 \\
\pm 0.87\end{array}$ & $\begin{array}{l}2.52 \\
\pm 0.77\end{array}$ & $\begin{array}{l}2.49 \\
\pm 0.99\end{array}$ & $\begin{array}{l}2.32 \pm \\
0.96\end{array}$ & $\begin{array}{l}2.45 \pm \\
0.91\end{array}$ \\
\hline & 45 & $\begin{array}{l}\text { Much of what I have to learn } \\
\text { seems relevant to a career in } \\
\text { healthcare }\end{array}$ & $\begin{array}{l}2.97 \pm \\
0.676\end{array}$ & $\begin{array}{l}2.96 \pm \\
0.61\end{array}$ & $\begin{array}{l}2.97 \pm \\
0.70\end{array}$ & $\begin{array}{l}3.00 \pm \\
0.66\end{array}$ & $\begin{array}{l}2.69 \pm \\
0.68\end{array}$ & $\begin{array}{l}2.91 \pm \\
0.67\end{array}$ \\
\hline \multirow[t]{5}{*}{ SPA } & 11 & $\begin{array}{l}\text { The atmosphere is relaxed } \\
\text { during clinical teaching }\end{array}$ & $\begin{array}{l}2.55 \\
\pm 1.00\end{array}$ & $\begin{array}{l}2.52 \pm \\
0.81\end{array}$ & $\begin{array}{l}2.56 \pm \\
0.86\end{array}$ & $\begin{array}{l}2.36 \\
\pm 1.08\end{array}$ & $\begin{array}{l}2.48 \pm \\
0.96\end{array}$ & $\begin{array}{l}2.49 \pm \\
0.95\end{array}$ \\
\hline & 12 & The course is well timetabled & $\begin{array}{l}2.06 \\
\pm 1.08\end{array}$ & $\begin{array}{l}1.33 \pm \\
1.18\end{array}$ & $\begin{array}{l}1.53 \\
\pm 1.21\end{array}$ & $\begin{array}{l}1.74 \\
\pm 1.20\end{array}$ & $\begin{array}{l}1.80 \pm \\
1.22\end{array}$ & $\begin{array}{l}1.70 \\
\pm 1.20\end{array}$ \\
\hline & 17 & $\begin{array}{l}\text { Cheating is a problem in this } \\
\text { course }\end{array}$ & $\begin{array}{l}1.75 \\
\pm 1.07\end{array}$ & $\begin{array}{l}1.96 \\
\pm 1.30\end{array}$ & $\begin{array}{l}2.34 \pm \\
1.12\end{array}$ & $\begin{array}{l}1.78 \\
\pm 1.20\end{array}$ & $\begin{array}{l}1.95 \\
\pm 1.15\end{array}$ & $\begin{array}{l}1.94 \\
\pm 1.18\end{array}$ \\
\hline & 23 & $\begin{array}{l}\text { The atmosphere is relaxed } \\
\text { during lectures }\end{array}$ & $\begin{array}{l}2.53 \\
\pm 0.87\end{array}$ & $\begin{array}{l}2.44 \pm \\
0.87\end{array}$ & $\begin{array}{l}2.36 \pm \\
0.90\end{array}$ & $\begin{array}{l}2.31 \pm \\
0.97\end{array}$ & $\begin{array}{l}2.59 \pm \\
1.01\end{array}$ & $\begin{array}{l}2.45 \\
\pm 0.93\end{array}$ \\
\hline & 30 & $\begin{array}{l}\text { There are opportunities for me } \\
\text { to develop interpersonal skills }\end{array}$ & $\begin{array}{l}2.95 \pm \\
0.81\end{array}$ & $\begin{array}{l}2.63 \pm \\
0.82\end{array}$ & $\begin{array}{l}2.65 \pm \\
0.72\end{array}$ & $\begin{array}{l}2.61 \pm \\
1.01\end{array}$ & $\begin{array}{l}2.49 \pm \\
1.10\end{array}$ & $\begin{array}{l}2.66 \pm \\
0.92\end{array}$ \\
\hline
\end{tabular}




\begin{tabular}{|c|c|c|c|c|c|c|c|c|}
\hline & 33 & $\begin{array}{l}\text { I feel comfortable in teaching } \\
\text { sessions socially }\end{array}$ & $\begin{array}{l}2.65 \pm \\
0.65\end{array}$ & $\begin{array}{l}2.66 \pm \\
0.83\end{array}$ & $\begin{array}{l}2.73 \\
\pm 0.69\end{array}$ & $\begin{array}{l}2.66 \pm \\
0.85\end{array}$ & $\begin{array}{l}2.48 \pm \\
0.81\end{array}$ & $\begin{array}{l}2.63 \pm \\
0.78\end{array}$ \\
\hline & 34 & $\begin{array}{l}\text { The atmosphere is relaxed } \\
\text { during seminars/tutorials }\end{array}$ & $\begin{array}{l}2.73 \\
\pm 0.75\end{array}$ & $\begin{array}{l}2.56 \pm \\
0.87\end{array}$ & $\begin{array}{l}2.42 \pm \\
0.88\end{array}$ & $\begin{array}{l}2.51 \pm \\
1.01\end{array}$ & $\begin{array}{l}2.51 \\
\pm 0.93\end{array}$ & $\begin{array}{l}2.55 \pm \\
0.89\end{array}$ \\
\hline & 35 & $\begin{array}{l}\text { I find the experience } \\
\text { disappointing }\end{array}$ & $\begin{array}{l}2.12 \\
\pm 0.93\end{array}$ & $\begin{array}{l}2.66 \pm \\
0.83\end{array}$ & $\begin{array}{l}2.34 \pm \\
0.98\end{array}$ & $\begin{array}{l}2.19 \pm \\
1.05\end{array}$ & $\begin{array}{l}2.10 \pm \\
1.06\end{array}$ & $\begin{array}{l}2.17 \pm \\
1.04\end{array}$ \\
\hline & 36 & I am able to concentrate well & $\begin{array}{l}2.75 \pm \\
0.79\end{array}$ & $\begin{array}{l}2.44 \pm \\
0.97\end{array}$ & $\begin{array}{l}2.63 \pm \\
0.64\end{array}$ & $\begin{array}{l}2.66 \\
\pm 0.83\end{array}$ & $\begin{array}{l}2.39 \\
\pm 1.00\end{array}$ & $\begin{array}{l}2.57 \pm \\
0.87\end{array}$ \\
\hline & 42 & $\begin{array}{l}\text { The enjoyment outweighs the } \\
\text { stress of studying medicine }\end{array}$ & $\begin{array}{l}2.50 \\
\pm 1.16\end{array}$ & $\begin{array}{l}2.18 \pm \\
1.10\end{array}$ & $\begin{array}{l}2.11 \pm \\
1.11\end{array}$ & $\begin{array}{l}2.21 \\
\pm 1.25\end{array}$ & $\begin{array}{l}2.33 \\
\pm 1.12\end{array}$ & $\begin{array}{l}2.27 \\
\pm 1.15\end{array}$ \\
\hline & 43 & $\begin{array}{l}\text { The atmosphere motivates me } \\
\text { as a learner }\end{array}$ & $\begin{array}{l}2.47 \\
\pm 1.01 \\
\end{array}$ & $\begin{array}{l}2.37 \\
\pm 1.02 \\
\end{array}$ & $\begin{array}{l}2.33 \pm \\
1.02\end{array}$ & $\begin{array}{l}2.36 \\
\pm 1.09 \\
\end{array}$ & $\begin{array}{l}2.16 \pm \\
1.04\end{array}$ & $\begin{array}{l}2.34 \\
\pm 1.04\end{array}$ \\
\hline & 50 & $\begin{array}{l}\text { I feel able to ask the questions } \\
\text { I want }\end{array}$ & $\begin{array}{l}2.68 \\
\pm 1.34\end{array}$ & $\begin{array}{l}2.57 \pm \\
1.38\end{array}$ & $\begin{array}{l}2.65 \pm \\
1.04\end{array}$ & $\begin{array}{l}2.42 \pm \\
1.35\end{array}$ & $\begin{array}{l}2.94 \pm \\
1.11\end{array}$ & $\begin{array}{l}2.66 \\
\pm 1.26\end{array}$ \\
\hline \multirow[t]{7}{*}{ SSSP } & 3 & $\begin{array}{l}\text { There is a good support system } \\
\text { for registrars who get stressed }\end{array}$ & $\begin{array}{l}2.05 \\
\pm 1.02 \\
\end{array}$ & $\begin{array}{l}1.97 \pm \\
1.21\end{array}$ & $\begin{array}{l}1.81 \pm \\
1.01\end{array}$ & $\begin{array}{l}1.56 \pm \\
1.02 \\
\end{array}$ & $\begin{array}{l}1.90 \\
\pm 1.05\end{array}$ & $\begin{array}{l}1.86 \pm \\
1.08\end{array}$ \\
\hline & 4 & $\begin{array}{l}\text { I am too tired to enjoy this } \\
\text { course }\end{array}$ & $\begin{array}{l}1.75 \\
\pm 0.99\end{array}$ & $\begin{array}{l}2.19 \pm \\
1.16\end{array}$ & $\begin{array}{l}1.88 \pm \\
1.10\end{array}$ & $\begin{array}{l}1.85 \\
\pm 1.17\end{array}$ & $\begin{array}{l}1.78 \pm \\
1.03\end{array}$ & $\begin{array}{l}1.89 \pm \\
1.10\end{array}$ \\
\hline & 14 & $\begin{array}{l}\text { I am rarely bored with this } \\
\text { course }\end{array}$ & $\begin{array}{l}2.32 \\
\pm 1.12 \\
\end{array}$ & $\begin{array}{l}2.00 \\
\pm 1.09\end{array}$ & $\begin{array}{l}1.78 \\
\pm 0.99\end{array}$ & $\begin{array}{l}2.09 \\
\pm 1.23\end{array}$ & $\begin{array}{l}2.06 \\
\pm 1.21\end{array}$ & $\begin{array}{l}2.06 \\
\pm 1.15\end{array}$ \\
\hline & 15 & $\begin{array}{l}\text { I have good friends in this } \\
\text { course }\end{array}$ & $\begin{array}{l}3.36 \pm \\
0.69\end{array}$ & $\begin{array}{l}3.40 \pm \\
0.84\end{array}$ & $\begin{array}{l}3.37 \pm \\
0.80\end{array}$ & $\begin{array}{l}3.34 \\
\pm 0.86\end{array}$ & $\begin{array}{l}3.10 \pm \\
0.90\end{array}$ & $\begin{array}{l}3.31 \pm \\
0.82\end{array}$ \\
\hline & 19 & My social life is good & $\begin{array}{l}2.51 \pm \\
0.95\end{array}$ & $\begin{array}{l}2.78 \\
\pm 1.11\end{array}$ & $\begin{array}{l}2.44 \\
\pm 1.20\end{array}$ & $\begin{array}{l}2.48 \pm \\
1.30\end{array}$ & $\begin{array}{l}2.58 \\
\pm 1.11\end{array}$ & $\begin{array}{l}2.56 \\
\pm 1.14\end{array}$ \\
\hline & 28 & I seldom feel lonely & $\begin{array}{l}2.43 \\
\pm 0.81 \\
\end{array}$ & $\begin{array}{l}2.24 \pm \\
1.14 \\
\end{array}$ & $\begin{array}{l}2.07 \\
\pm 1.20 \\
\end{array}$ & $\begin{array}{l}2.26 \\
\pm 1.13 \\
\end{array}$ & $\begin{array}{l}2.21 \pm \\
1.16 \\
\end{array}$ & $\begin{array}{l}2.25 \\
\pm 1.09\end{array}$ \\
\hline & 46 & My accommodation is pleasant & $\begin{array}{l}3.30 \pm \\
0.68\end{array}$ & $\begin{array}{l}3.01 \\
\pm 1.11\end{array}$ & $\begin{array}{l}3.15 \\
\pm 0.88\end{array}$ & $\begin{array}{l}3.31 \\
\pm 0.79\end{array}$ & $\begin{array}{l}3.05 \pm \\
1.04\end{array}$ & $\begin{array}{l}3.16 \\
\pm 0.91\end{array}$ \\
\hline
\end{tabular}

(SPL - students' perception of learning, SPT - students' perception of teachers, SASP - students' perception academic selfperceptions, SPA - students' perception of atmosphere, SSSP - students' social self-perceptions.)

\section{DISCUSSION}

Educational environment is a major determinant of the success of curriculum. There is an association between the educational climate and student's achievement, satisfaction, and performance. ${ }^{20}$ Hence, physiotherapy schools should strive to offer the best possible educational environment and teaching - learning experience to encourage students to perform to their full potential. Students form a significant part in the evaluation and enhancement of the quality of this learning experience. The DREEM inventory allows us to identify the areas of concern in the educational environment.

The total DREEM mean score was $122.66 \pm 17.39$, indicating that the perception of the learning environment among physiotherapy students was 'more positive than negative.' Our study showed that the students reported perception of learning, perception of atmosphere, and academic self-perception as positive. The domain perception of teachers was reported to be 'moving in the right direction.' The domain of students' social self-perceptions was 'not too bad.' Edgren et al. (2010) indicated that results from the overall perception of the educational environment and the subscales may mask the presence of explicit educational problems, and that analysis on an item level is necessary. ${ }^{21}$ Hence, item-wise analysis was done to fully explore areas of strength and weakness. 
Students' perception of learning: The highest mean scores were reported for this domain. Items with the highest scores were 'students' awareness of objectives of the course' and 'focus on long-term learning as compared to short-term.' Items that scored less than 2 points pertained to 'factual learning process' and 'teacher-centered learning.' Many institutions globally report similar concerns. ${ }^{16}$ In the current undergraduate physiotherapy program, formative and summative assessment is the major evaluation process undertaken in each academic year. This leads to a focus on factual and passive learning as students have to perform well and score higher grades. A problem-based evaluation might provide students with stimulating opportunities for learning and thereby build their confidence. Teaching-learning methodology has to move away from the memorization of facts and passive learning to promote self-directed and active learning that engage students. ${ }^{16,21-22}$ Lowest scores were observed in interns who mainly focus on bedside teaching, or work in an outpatient department with only a few hours a day spent in lectures.

Students' perception of teachers: The highest scores were reported among final year physiotherapy students. Items in this domain that scored less than 2 points pertained to 'authoritarian teachers' and 'inability to deliver constructive criticism.' This is attributed to the traditional styles of teaching in our institution. In an Indian setup, a teacher has to play multiple roles in academic as well as clinical setup. They are engaged in different activities like classroom instruction, clinical teaching and providing treatment to the patients at the same time. Students observe and emulate their behavior and attitude. They look up to teachers as a role model. Teachers have to inculcate lifelong professional values and integrity in the students and help to groom their skills. In this process, teachers should be aware that respecting the student is critical to the learning process. ${ }^{2}$ Excessive harsh criticism or absence of any feedback is discouraging and damaging to students' self-confidence. Hence, mutual respect and proper dignity should be maintained. With the current emphasis on self-directed and life-long learning, teachers are no longer the only providers of information, but they should facilitate the acquisition of attitudes and skills necessary for learning. ${ }^{16}$ This will assist in improvement of the educational climate.

Students' academic self-perception: The mean score in this domain was $21.23 \pm 3.72$, which is low compared to other domains with all items between 2 to 3 . Academic self-perception is associated with the ability of the student to cope with the academic workload. Many studies have reported low scores in this domain, suggesting that curriculum overload is a common problem, regardless of whether the curriculum is traditional or innovative. ${ }^{16,21}$ It also depends on the individual personality, learning styles, and coping strategies which should be targeted to develop the academic self-perception.

Students' perceptions of atmosphere: The mean score in this domain was $28.56 \pm 5.64$, which reflected a more positive atmosphere. Students perceived that the institution had a healthy atmosphere to foster interpersonal skills. The ability to solve problems and socially comfortable teaching sessions were appreciated the most. Items in this domain that scored less than two points pertained to 'poor timetabling and scheduling of lectures.' In the Indian scenario, teachers are not only academicians but also clinicians. At times, the clinical workload and sudden emergency patient calls take a toll on the academic schedule as faculty have to prioritize clinical work over the teaching sessions. This may lead to cancellation of the lectures and affect the teaching schedule. A well-planned academic calendar and proper reliever arranged during the emergency duties may benefit the teaching program.

Students' social self-perception: The lowest mean score of $17.13 \pm 3.08$ was observed in this domain as compared to other subscales. Items in this domain that scored less than 2 points were 'poor support system for students who get stressed' and 'tiredness during the course.' Students reported that they were happy with their friends and had a good social life. Student mentoring, as a means of providing academic and social support, should be implemented to tackle these issues. Mentoring programs could help to generate more near-peer mentors for senior students. Curriculum planners could consider ways to make the curriculum less bulky and more innovative, engaging, and meaningful so as to avoid student boredom and tiredness. ${ }^{23}$

In our study, there was a decrease in the perceived educational environment among interns. Rothoff et al. (2011) stated that educational environment is not exclusively dependent on educational delivery but also on individual characteristics of the students. ${ }^{24}$ As the students progress further in the academic years, they become more autonomous, critical, and self-directed. These variables affect their learning outcomes and perception of educational climate.

The overall DREEM scores for a medical school in India was reported as 107.44/200. ${ }^{25} \mathrm{~A}$ recently published study conducted in physiotherapy students reported the overall DREEM score of 150/200 and rated the educational environment as "more positive than negative". Two items that obtained less scores were authoritarian teachers and teaching with an overemphasis on factual learning which is consistent with our study. ${ }^{26}$

A limitation of this study is the use of a questionnaire with predetermined choices to evaluate educational environment, and some factors that may impact the environment in our institution may have been left out. Secondly, for this type of study, a qualitative method may have covered all the aspects of the educational environment that were overlooked in our study. Every institute provides different learning experience to the learners especially where there is no statutory control over the educational program.

( The Internet Journal of Allied Health Sciences and Practice, 2019 
The educational environment is dependent on multiple factors that are specific to the individual institution. It represents the primary assessment of students' perception of the educational environment at this institution that can be considered as baseline to monitor the effects of curricular change or reforms in future. It provides a basis for implementing modifications, helps in identification of weaknesses in curricula and thus optimizing the educational environment. 27,28

\section{CONCLUSION}

This study showed that all undergraduate physiotherapy students perceived their educational environment positively. However, some areas require remedial measures in order to enhance the educational experience. The greatest difficulty was with students' social self-perception. Specifically, students gave the lowest scores to the institutional support system, burdensome course content and factual learning. Inclusion of problem-based learning will give a more practical oriented approach emphasizing on active learning process which might provide students with stimulating opportunities for learning. ${ }^{29}$ Student support and mentorship programs should be implemented to strengthen interpersonal relation among peers. Continuous teaching learning workshop for the educators will help to enhance and enrich the learning environment.

\section{REFERENCES:}

1. Roff S, McAleer S. What is educational climate? MedTeach 2001; 23: 333-334.

2. Genn JM. AMEE Medical education guide no. 23 (part2): curriculum, environment, climate, quality and changein medical education. A unifying perspective. Med Teach. 2001; 23: 445-454.

3. International standards in medical education: assessment and accreditation of medical schools' - educational programmes. A WFME position paper. The Executive Council, The World Federation for Medical Education. Med Educ. 1998;32(5):549-558.

4. Genn JM. AMEE Medical Education Guide No. 23 (Part 1): Curriculum, environment, climate, quality and change in medical education-a unifying perspective. Med Teach 2001; 23:337-44.

5. Harden RM. The learning environment and the curriculum. Med Teach. 2001;23(4):335-336.

6. Bassaw B, Roff S, McAleer S, Roopnarinesingh S, De Lisle J, Teelucksingh S, et al. Students' perspectives on the educational environment, Faculty of Medical Sciences, Trinidad. Med Teach. 2003;25(5):522-26.

7. Panhale VP, Bellare B. Evidence-based practice among physiotherapy practitioners in Mumbai, India. Educ Health 2015; 28:154-5.

8. Mojaddidi MA, Khoshhal KI, Habib F, Shalaby S, El-Bab ME, Al-Zalabani AH. Reassessment of the undergraduate educational environment in College of Medicine, Taibah University, AlmadinahAlmunawwarah, Saudi Arabia. Med Teach. 2013. 35(Suppl 1): S39-S46.

9. Lo C. How student satisfaction factors affect perceived learning. Journal of the Scholarship of Teaching and Learning.2010;10:47-54.

10. Roff S, McAleer S, Harden RM, et al. Development and validation of the Dundee Ready Education Environment Measure (DREEM). Med Teach. 1997; 19:295-299.

11. Roff S, McAleer S, Skinner A. Development and validation of an instrument to measure the postgraduate clinical learning and teaching educational environment for hospital-based junior doctors in the UK. Med Teach. 2005; 27:326331.

12. Strand P, Sjöborg K, Stalmeijer R, Wichmann-Hansen G, Jakobsson U, Edgren G. Development and psychometric evaluation of the Undergraduate Clinical Education Environment Measure. Med Teach. 2013; 35:1014-1026.

13. Whittle SR, Whelan B, Murdoch-Eaton DG. DREEM and beyond: studies of the educational environment as a means for its enhancement. Educ Health. 2007; 20:7.

14. Denz-Penhey $\mathrm{H}$, Murdoch JC. A comparison between findings from the DREEM questionnaire and that from qualitative interviews. Med Teach. 2009; 31:449-453.

15. Soemantri D, Herrera C, Riquelme A. Measuring the educational environment in health professions studies: a systematic review. Med Teach. 2010; 32:947-952.

16. Veerapen K, McAleer S. Students' perception of the learning environment in a distributed medical programme. Med Educ Online. 2010;15.

17. Roff S, McAleer S, Harden RM, et al. Development and validation of the Dundee Ready Education Environment Measure (DREEM). Med Teach. 1997; 19:295-299.

18. McAleer S, Roff S. A practical guide to using the Dundee Ready Education Environment Measure (DREEM). In: Genn $J M$, ed. Curriculum, environment, climate, quality and change in medical education: a unifying perspective. Dundee, UK: Association of Medical Education in Europe; 2001. p 29-33.

19. Miles S, Swift L, Leinster $S$ The Dundee Ready Education Environment Measure (DREEM): A review of its adoption and use. Medical Teacher. 2010 34:9, e620-e634. 
20. Genn JM, Harden RM. What is medical education here really like? Suggestions for action research studies of climates of medical education environments. Medical Teacher 1986; 8(20): 111-24.

21. Edgren G, Haffling AC, Jakobsson U, McAleer S, Danielsen N. Comparing the educational environment (as measured by DREEM) at two different stages of curriculum reform. Med Teach. 2010;32: e233-e238.

22. Kohli V, Dhaliwal U. Medical students' perception of the educational environment in a medical college in India: a crosssectional study using the Dundee Ready Education Environment questionnaire. J EducEval Health Prof. 2013; 30:5.

23. Arzuman H, Yusoff MS, Chit SP. Big Sib. Students' perceptions of the educational environment at the School of Medical Sciences, UniversitiSains Malaysia, using Dundee Ready Educational Environment Measure (DREEM) Inventory. Malays J Med Sci. 2010; 17:40-47.

24. Rotthoff T, Ostapczuk MS, De Bruin J, Decking U, Schneider M, RitzTimme S. Assessing the learning environment of a faculty: psychometric validation of the German version of the Dundee Ready Education Environment Measure with students and teachers. Med Teach. 2011; 33:624-636.

25. Mayya SS, Roff S: Students' Perceptions of Educational Environment: A Comparison of Academic Achievers and Under-Achievers at Kasturba Medical College, India. Education for Health 2004, 17:280-291.

26. Palmgren $P$, Lindquist I, Sundberg T, Nilsson G, Laksov K. Exploring perceptions of the educational environment among undergraduate physiotherapy students. International Journal of Medical Education. 2014; 5:135-146.

27. Hammond SM, O'Rourke M, Kelly M, Bennett D, O'Flynn S. A psychometric appraisal of the DREEM. BMC Med Educ. 2012; 12:2.

28. Edgren G, Haffling AC, Jakobsson U, McAleer S, Danielsen N. Comparing the educational environment (as measured by DREEM) at two different stages of curriculum reform. Med Teach. 2010; 32(6): e233-e238.

29. Qin Y, Wang Y, Floden RE. The effect of problem-based learning on improvement of the medical educational environment: a systematic review and meta-analysis. Med Princ Pract 2016; 25:525-32. 